\title{
Follow-up searches for ultra-high energy neutrinos from transient astrophysical sources with the Pierre Auger Observatory
}

\author{
Michael Schimp $^{* a}$ for the Pierre Auger Collaboration ${ }^{\dagger b}$ \\ ${ }^{a}$ University of Wuppertal, Wuppertal, Germany \\ ${ }^{b}$ Observatorio Pierre Auger, Av. San Martín Norte 304, 5613 Malargüe, Argentina \\ E-mail: auger_spokespersons@fnal.gov \\ Full author list: http://www.auger.org/archive/authors_icrc_2019.html
}

\begin{abstract}
We present searches for ultra-high energy (UHE) neutrinos $(>0.1 \mathrm{EeV})$ with the Pierre Auger Observatory, following the detections of various types of transient astrophysical sources. These include binary black hole (BBH) mergers, detected via gravitational waves (GWs) by the LIGO and Virgo detectors. The results of the searches for a flux of UHE neutrinos from the BBH mergers combined are reported and include events from the currently ongoing third observing run of the LIGO and Virgo GW detectors. Another source of interest in the context of multimessenger (MM) astrophysics is TXS 0506+056, a blazar that was found to emit both photons and neutrinos in a correlated manner, but at lower energies than accessible with the Pierre Auger Observatory. Follow-up searches for UHE neutrinos were performed during periods of increased emission of high-energy photons and neutrinos from TXS 0506+056 and the corresponding results are reported.
\end{abstract}

36th International Cosmic Ray Conference - ICRC2019

24 July - 1 August, 2019

Madison, Wisconsin, USA

* Speaker.

${ }^{\dagger}$ for collaboration list see PoS(ICRC2019)1177 


\section{Introduction}

The Pierre Auger Observatory near Malargüe in the Province of Mendoza, Argentina, is the largest and most precise UHE cosmic-ray detector in the world. The Observatory relies on several different detection systems, one of which is the surface detector (SD), a triangular grid consisting of 1660 water-Cherenkov detectors (WCDs) with a spacing of $1.5 \mathrm{~km}$. Overall, the SD covers an area of $3000 \mathrm{~km}^{2}$ and has an average altitude of $1400 \mathrm{~m}$ a.s.l., corresponding to a vertical atmospheric depth of $875 \mathrm{~g} / \mathrm{cm}^{2}$. Each WCD is filled with 12 tons of ultra-pure water. When charged particles in extensive air showers (EAS) traverse this water, Cherenkov radiation is emitted and detected using photomultiplier tubes (PMTs). These PMT signals are used to determine EAS properties such as the arrival direction and energy of the primary particle. The Pierre Auger Observatory has been taking data since the beginning of 2004. Its components are described in detail in [1].

Searches for UHE neutrinos and photons with the Pierre Auger Observatory are described in these proceedings [2,3]. UHE neutrinos and photons are thought to originate from UHE cosmic rays, being produced either at UHE cosmic-ray sources or during their propagation through the Universe [4-8]. Therefore, the established UHE neutrino and photon search procedures are used as an element in MM searches by following up astrophysical observations made by other instruments.

As neutrinos and photons are electrically neutral, they are not deflected in magnetic fields, and their arrival directions thus point back to their sources. In addition, neutrinos are only subject to the weak interaction, leading to almost no attenuation and therefore to virtually no neutrino horizon as opposed to photons, which interact also electromagnetically and therefore have only a finite effective path length. This length is much shorter than the luminosity distances to the sources followed up in this work, $d>300 \mathrm{Mpc}$ [9]. Therefore, this work will focus on UHE neutrinos.

One previous example of a MM follow up with the Pierre Auger Observatory is the search for UHE neutrinos from the first ever observed binary neutron star merger, which was detected as GW170817 via GWs by the LIGO Scientific Collaboration and the Virgo Collaboration (LVC) [10]. Additionally, a wide range of signals from this object was found throughout the electromagnetic spectrum [10,11], leading to a rich set of astronomical and astrophysical insights, and making it a milestone of MM astronomy. At the time of the merger, the source of GW170817 was located about $2^{\circ}$ below the horizon at the site of the Pierre Auger Observatory. This direction is in the most sensitive part of the field of view of the Pierre Auger Observatory, the so-called Earth-skimming (ES) region. Accordingly, Auger provided the strongest constraints on the UHE neutrino fluence in the period of $1000 \mathrm{~s}$ symmetrically around the merger, which is the a priori fixed search period for prompt emission from this source [12].

Here we focus on two types of potential transient UHE neutrino sources. The first are binary black hole mergers that were found with the LVC GW detectors. The LIGO Scientific Collaboration discovered BBH mergers with the very first directly detected GWs in 2015, initiating the era of GW astronomy [13,14]. An observation of the BBH mergers by other means has not yet been successful, despite many searches for a signal by a large number of instruments. The other source type is a specific single source, the blazar TXS 0506+056. The IceCube neutrino observatory detected a high-energy $(\sim 0.3 \mathrm{PeV})$ neutrino from the direction of TXS 0506+056 in 2017, leading to the so-called IceCube-170922A alert that allowed other observatories to perform followup observations [15]. TXS 0506+056 was in a $\gamma$-ray flaring state at the time of the detection of the 
high-energy neutrino [16]. The coincidence of the neutrino and the $\gamma$-ray flare was found not to be accidental with a significance of $3 \sigma$ [15]. Additionally, and statistically even more significantly, a $3.5 \sigma$ excess of neutrinos from this source was found in archival data [17]. Naturally, these findings constitute another important step in the development of MM astronomy.

In the following sections, the follow-up search methods, parameters, and results for both source types are described and discussed.

\section{Searches for ultra-high energy neutrinos from binary black hole mergers}

For the 21 events detected and published by the LVC until 2019-06-02 that are most likely originating from BBH mergers [18-29], follow-up searches for UHE neutrinos with the Pierre Auger Observatory have been performed as described below. For the searches, events detected with Auger were only considered to be coincident with a BBH merger if they were detected within 24 hours after the merger and came from within the most probable (90\% C.L.) localization quantile in the sky, $\Omega_{90}$, which is provided by the LVC. Using the established UHE neutrino search methods [2], no candidates have been found in such a coincidence with any of the BBH mergers.

In contrast to previous searches, for which follow-up results were expressed in terms of flux or fluence from a single specific source during two fixed search periods, here, the hypothetical fluxes of UHE neutrinos from all followed up BBH mergers are combined as follows. As very little is known about the sources, it is assumed that each has the same isotropic UHE neutrino luminosity as a function of time after the merger. This function, denoted $L\left(t-t_{0}\right)$, with the merger time $t_{0}$, has no a priori constraints. In addition, the differential UHE neutrino flux from each source follows the commonly assumed power law $\frac{\mathrm{d} \Phi}{\mathrm{d} E_{v}} \propto E_{v}^{-2}$, with the neutrino energy $E_{v}$. These assumptions, together with observational properties, like the localization probability of each source as a function of its direction in the sky, and the neutrino identification efficiency of Auger, allows us to quantify the time-dependent sensitivity to the combined UHE neutrino flux from the sources. As a specific application of this sensitivity, considering that no UHE neutrinos have been found, a 90\% C.L. upper bound on $L\left(t-t_{0}\right)$ is calculated and discussed below.

For determining the sensitivity, a key quantity is the effective area $A_{\text {eff }}\left(E_{v}, \theta, t-t_{0}\right)$ of the Observatory, as introduced in [30]. It is proportional to the geometrical area of the SD and to the efficiency of the neutrino detection and identification as a function of $E_{v}$ and zenith angle $\theta$. The explicit dependence of $A_{\text {eff }}$ on time originates from minor changes in the status of the SD that can lead to SD stations not being considered for data analysis during certain periods of time, changing the Observatory's used geometrical area. The relationship between the number of identified neutri$\operatorname{nos} N_{v}$ from the considered sources with index $s$, the universal isotropic UHE neutrino luminosity of each source $L\left(t-t_{0}\right)$, and the effective area $A_{\text {eff }}\left(E_{v}, \theta, t-t_{0}\right)$ can be written in integral form as:

$$
N_{v}=\int_{t_{0}}^{t_{0}+24 \mathrm{~h}} \iint_{\Omega_{90}} \int_{0}^{\infty} \sum_{s} \frac{L(t)}{d_{s}^{2}} P_{s}(\delta, \alpha) E_{v}^{-2} A_{\mathrm{eff}}\left(E_{v}, \theta(\delta, \alpha, t), t\right) \mathrm{d} E_{v} \mathrm{~d} \Omega \mathrm{d} t
$$

where the BBH mergers as sources with index $s$ have a probability density per solid angle $P_{s}(\delta, \alpha)$ to be localized at a certain direction and have the best-fit luminosity distance $d_{s}$ given by the LVC. 


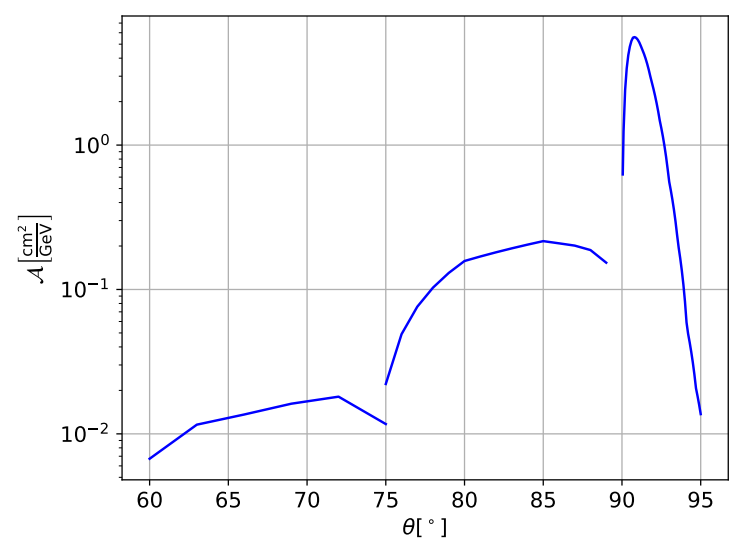

Figure 1: $\mathcal{A}(\theta, t)$ averaged over the year 2016

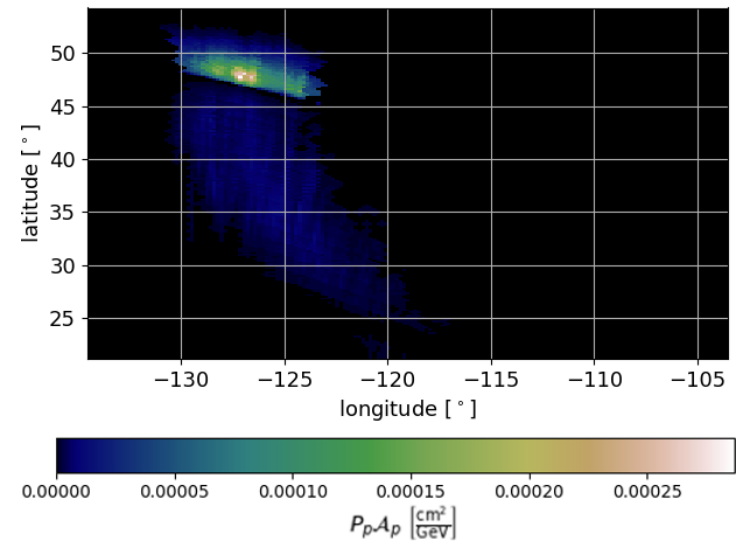

Figure 2: Localization probability per pixel $P_{p}$ times energy-spectrum weighted effective area $\mathcal{A}_{p}$ in equatorial coordinates for the BBH merger GW event GW170608, 20h40m after the merger

The energy integral $\mathcal{A}(\theta, t)=\int_{0}^{\infty} E_{v}^{-2} A_{\mathrm{eff}}\left(E_{v}, \theta, t\right) \mathrm{d} E_{v}$ is evaluated separately. Figure 1 shows the average of $\mathcal{A}(\theta, t)$ for the year 2016, which is the benchmark time period for effective areas used in [2]. The zenith-angle dependence of the sensitivity is notable and exhibits a strong peak at $\theta=90.8^{\circ}$.

For each BBH merger, the localization probability of each source $s$ is provided by the LVC for a set of discrete equally sized pixels that are fixed in equatorial coordinates. The pixels in the $90 \%$ C.L. region $\Omega_{90}$ will be indexed with $p$ in the following, and accordingly the probability for the source $s$ to be localized within a pixel $p$ is $P_{p, s}$. For the calculation in terms of pixels, the coordinates $(\delta, \alpha)$ from Equation 2.1 are converted into the pixels $p$, while the directional integration is converted into a sum over $p$. For each source $s$, the pixels are smaller than the mean point spread function of a reconstructed neutrino with Auger. Therefore, the pixel-wise localization probability provided by LIGO is sufficiently precise for the purpose of this analysis.

Analogously to the direction dependence of $P_{p, s}, \mathcal{A}$ is determined for each pixel and source individually, and in this formulation denoted $\mathcal{A}_{p, s}$. Figure 2 shows an example of $P_{p, s} \cdot \mathcal{A}_{p, s}$ in equatorial coordinates for the source GW170608 at $t-t_{0}=74400 \mathrm{~s}$. A diffuse structure with two distinct regions can be recognized. The bright pixels in the North, representing a large value for $P_{p, s} \cdot \mathcal{A}_{p, s}$, are below the local horizon of Auger and therefore part of the ES angular region $\left(90^{\circ}<\theta<95^{\circ}\right)$, where the sensitivity to neutrinos is large. The much fainter pixels further south belong to directions above the local horizon, where the sensitivity to neutrinos is much lower [2].

Evaluating the time-dependent quantities from Equation 2.1 in time bins $i$ with a width $\Delta t$, the following relation between the number of neutrinos per time bin, $N_{v, i}$, and the corresponding universal isotropic UHE luminosity of the sources in that time bin, $L_{i}$, holds:

$$
N_{v, i}=L_{i} \Delta t \sum_{s} \frac{\sum_{p} P_{p, s} \mathcal{A}_{p, s, i}}{d_{s}^{2}}
$$

No neutrinos were found with Auger during any 24-hour time interval after the mergers. Thus, 


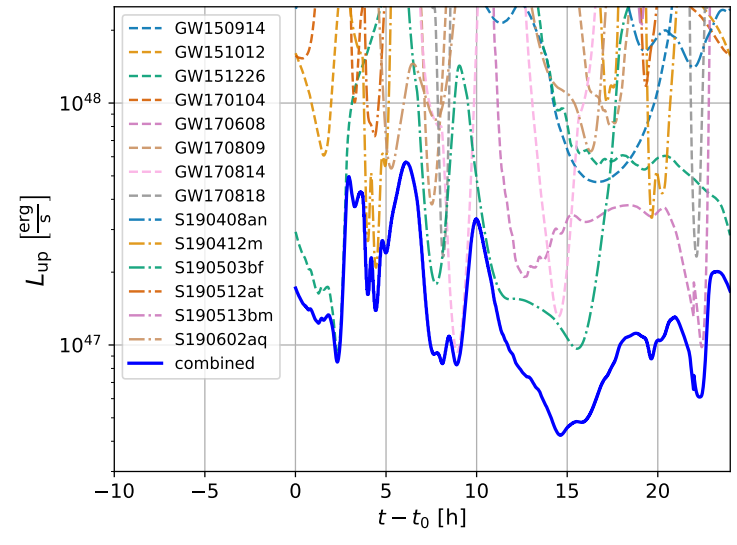

Figure 3: Solid line: upper limit on the timedependent universal isotropic neutrino luminosity; dashed (dash-dotted) lines: individual single-source contributions from the first two (third) LIGO/Virgo observing runs; sources not indicated here are above the $L_{\mathrm{up}}$-range of the Figure

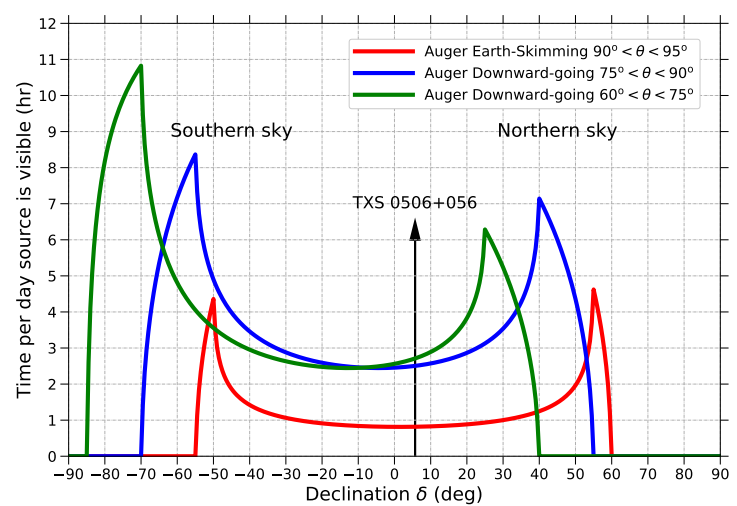

Figure 4: Lines: time per day that a given declination lies in the different neutrino search zenith regions of Auger; arrow: declination of TXS 0506+056

as an application, the $90 \%$ C.L. upper bound on the isotropic luminosity, $L_{\mathrm{up}, i}$, for all time bins $i$ is derived in the following. The $90 \%$ C.L. upper bound on the number of UHE neutrinos identified in the 24 hours after all mergers combined is $N_{\text {up }, v, \text { tot }}=2.44$. The value is the $90 \%$ C.L. sensitivity to a Poisson signal when observing zero events with a background expectation of zero [31], which matches the given situation to a very good approximation. The time bins are chosen to have a width of $\Delta t=1 \mathrm{~s}$, so the results are calculated for 86400 of these bins, comprising 24 hours in total. This precision in time is high enough to assume that the results for $L_{\mathrm{up}, i}$ will very precisely match the upper bound on the true continuous function $L\left(t-t_{0}\right)$.

Using the considerations and assumptions above, the relation $N_{\mathrm{up}, v, \text { tot }}=2.44=\sum_{i} N_{\mathrm{up}, v, i}=$ $\frac{24 \mathrm{~h}}{\Delta t} N_{\text {up }, v, i} \Rightarrow \frac{N_{\text {up }, v, i}}{\Delta t}=\frac{2.44}{86400 \mathrm{~s}}$ holds, where $N_{\text {up }, v, i}$ is the upper bound on the identified number of neutrinos per time bin $i$. The assumption that it is the same for all time bins was used here, since both the observed number of detected UHE neutrinos and, to a very good approximation, the expected number of background events are the same (zero) for all time bins. Equation 2.2 can thus be solved for the upper bound on the isotropic luminosity in the time bin $i$ :

$$
L_{\mathrm{up}, i}=\frac{2.44}{86400 \mathrm{~s}}\left(\sum_{s} \frac{\sum_{p} P_{p, s} \mathcal{A}_{p, s, i}}{d_{s}^{2}}\right)^{-1} .
$$

The result for $L_{\mathrm{up}, i}$ is shown in Figure 3, where the contributions from the individual sources are indicated as well. For sources whose contribution to the combined sensitivity is more dominant, the corresponding lines in Figure 3 are lower. The universal isotropic luminosity, obtained by combining the sources, is constrained more strongly than the single-source luminosities. This shows that the described combination of sources substantially improves the sensitivity to the source class of BBH mergers as compared to single sources of this class. Still, the combined limit is fully dominated by a single source at some times, e.g. by GW151226 $\sim 2.5$ hours after the merger. 
It should be noted that if this analysis was performed with a longer follow-up search duration, the result would be periodic with a period of 1 sidereal day corresponding to the periodic motion of the field of view of Auger in equatorial coordinates. This, added to the fact that it has been the standard for previous searches [32], substantiates the choice of the 24 hours after each merger as the search duration. With further BBH mergers being detected in the future, the total exposure will increase and either a signal will be eventually detected, or the UHE neutrino emission of the sources can be constrained better over time.

\section{Searches for UHE neutrinos from the neutrino-emitting blazar TXS 0506+056}

For the blazar TXS 0506+056, the two follow-up search time intervals used in this work are 2014-10-19 - 2015-02-06, and 2017-03-22 - 2017-09-22. The earlier one corresponds to the time of the excess of neutrinos reported by IceCube [17], while the later one is motivated by one of the benchmark durations given by IceCube that represent the $\gamma$-ray flare during which the IceCube170922A high-energy neutrino was found [15]. No neutrinos have been found with Auger during these periods.

TXS 0506+056 is a well located source and the follow-up periods consist of whole days. Therefore, the exposure to it depends only on its declination and, analogous to the BBH merger follow-up searches, small temporary variations in the status of Auger. Figure 4 illustrates the time per day a given declination lies in the different neutrino search regions, where the declination of TXS 0506+056 is indicated.

By taking into account these visibility times and the respective effective areas, the overall sensitivity to the source over a given time interval is obtained. The fluxes corresponding to certain numbers of neutrinos identified with Auger during a given period of time can then be determined in the same way as for the BBH follow-up searches. In the following, we present the fluxes that would correspond to an expectation of one identified UHE neutrino with Auger during each of the mentioned search periods.

In Figure 5, this flux is shown for the first search period (2014-10-19 - 2015-02-06) together with the flux of the neutrino excess measured by IceCube and extrapolations of that measurement to higher energies [17]. These results are shown for different power laws assumed for the flux. The spectrum measured by Fermi-LAT during this time [33] lies mostly below the IceCube flux in this representation. One can see that the flux that would correspond to one UHE neutrino identified by Auger is larger than the extrapolation by IceCube in this $E^{2}$-weighted representation. The ratio between them is $\sim 20$ for a hard spectrum $\propto E^{-1.9}$. This difference originates largely from the fact that the source is visible only periodically. In particular, it lies in the ES region, the region with the largest sensitivity, for a fraction of only $\sim \frac{1}{25}$ of the time. For a spectrum $\propto E^{-2.3}$, this ratio is $\sim 2000$, indicating the lower sensitivity of Auger to softer spectra.

The results for the second search period (2017-03-22 - 2017-09-22) are summarized in Figure 6. It can be inferred that, in this $E^{2}$-weighted representation, the flux associated to the IceCube170922A alert event is very similar to the photon flux measured by Fermi-LAT in the GeV range [15, 16]. However, the flux that would correspond to one UHE neutrino identified with Auger in this period is $\sim 20$ times larger than that, again explainable by the periodic visibility of the source, which is not optimal when averaging over time. 


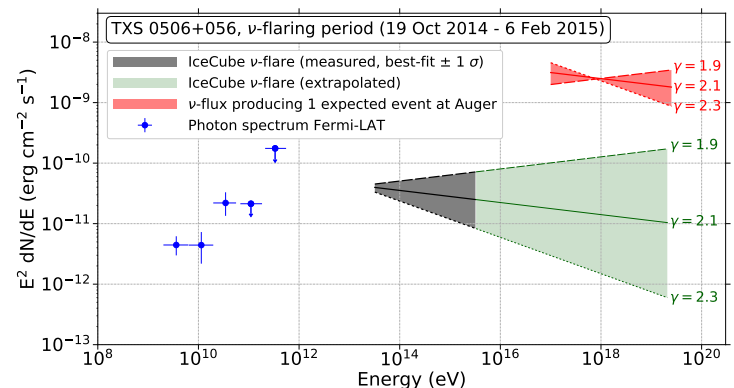

Figure 5: Fluxes associated with the TXS 0506+056 neutrino excess in 2014/2015; blue data points (arrows): measured (upper bounds on) photons from Fermi-LAT; lower shaded region in grey (pale green): IceCube measured (extrapolated) flux of neutrinos for a range of spectral indices; higher shaded region in red: UHE neutrino flux that would correspond to one neutrino being identified with Auger

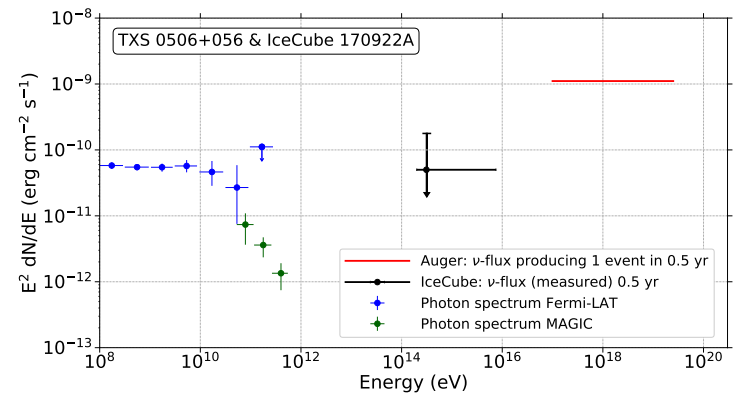

Figure 6: Fluxes from TXS 0506+056 in the time around IceCube-170922A; magenta, blue, and green data points (arrows): measured (upper bounds on) photons by the given instruments; black: neutrino flux of associated IceCube neutrino, assuming this event is the signal for 0.5 years; red: flux from this source that would correspond to one UHE neutrino detected with Auger during the same period

It should also be noted that the neutrino energy ranges regarded for Auger and IceCube in this analysis are about 3 orders of magnitude apart, meaning that both results are meaningful on their own in their respective energy domains.

\section{Summary and conclusions}

We presented the procedures and results of two different kinds of analyses of searches for UHE neutrinos from transient sources.

Searches for UHE neutrinos from BBH mergers as potential sources were combined and an upper limit obtained on a universal isotropic UHE neutrino luminosity as a function of time after the merger, that would apply to each of the sources. Approximately 14.6 hours after the merger, the combined sensitivity is maximal, and a universal isotropic source UHE neutrino luminosity $L_{\mathrm{up}}$ above $\sim 4.3 \cdot 10^{46} \frac{\mathrm{erg}}{\mathrm{s}}$ is excluded due to the non-observation of UHE neutrinos from the $90 \%$ C.L. BBH merger localization regions in the sky during the 24-hour periods after all mergers, under the assumption that the sources emit UHE neutrinos with a spectrum $\propto E_{v}^{-2}$.

The follow-up searches for UHE neutrinos from the direction of TXS 0506+056 were performed during two different time intervals, 2014-10-19 - 2015-02-06 and 2017-03-22 - 201709-22, motivated by a neutrino excess found with IceCube during the earlier one and a $\gamma$-ray flare with an associated high-energy neutrino alert lasting the later time interval. No UHE neutrinos have been observed in these searches by Auger. For comparison with the lower-energy IceCube neutrino fluxes from this source, the flux that would correspond to one UHE neutrino from TXS 0506+056 identified with Auger in each of the two follow-up periods has been calculated. For all considered periods and hypothetical energy spectral shapes, the $E^{2}$-weighted flux necessary to produce this one UHE neutrino has been found to be more than an order of magnitude larger than the flux estimations 
given by IceCube. While this indicates a smaller sensitivity to neutrinos from the TXS 0506+056 flares, explainable by the periodic visibility with interruptions, the fluxes apply to different energy ranges (TeV/PeV vs. EeV range) and complement each other well.

\section{References}

[1] A.Aab [Pierre Auger Collaboration], Nucl. Instrum. Meth. A 798 (2015) 172.

[2] F.Pedreira for the Pierre Auger Collaboration, POS ( ICRC2019) 979.

[3] J.Rautenberg for the Pierre Auger Collaboration, POS ( ICRC2019) 398.

[4] K.Greisen, Phys. Rev. Lett. 16 (1966) 748.

[5] G.T.Zatsepin, V.A.Kuz'min, JETP Lett. 4 (1966) 78.

[6] D.Hooper, A.Taylor, S.Sarkar, Astropart. Phys. 23 (2005) 11.

[7] M.Ave, N.Busca, A.V.Olinto, A.A.Watson, T.Yamamoto, Astropart. Phys. 23 (2005) 19.

[8] K.Kotera, D.Allard, A.V.Olinto, JCAP 2010 (2010) 013.

[9] M.Risse and P.Homola, Mod. Phys. Lett. A, 22 (2007) 749.

[10] B.P.Abbot et al., Astrophys. J. Lett. 848 (2017) L12.

[11] I.Arcavi et al., Nature 551 (2017) 64.

[12] A.Albert et al., Astrophys. J. Lett. 850 (2017) L35.

[13] B.P.Abbot [LIGO Scientific Collaboration and Virgo Collaboration], Phys. Rev. Lett. 116 (2016) 061102.

[14] B.P.Abbot [LIGO Scientific Collaboration and Virgo Collaboration], Phys. Rev. Lett. 116 (2016) 241103.

[15] IceCube Collaboration, Science 361 (2018) eaat1378.

[16] Y.T.Tanaka, S.Buson, D.Kocevski for the Fermi-LAT collaboration, Astronomer's Telegram 10791 (2017).

[17] IceCube Collaboration, Science 361 (2018) 147.

[18] B.P.Abbot [LIGO Scientific Collaboration and Virgo Collaboration], arXiv preprint (2018) arXiv:1811.12907.

[19] L.Singer [LIGO Scientific Collaboration and Virgo Collaboration], GCN Circular 24069 (2019).

[20] P.Shawhan [LIGO Scientific Collaboration and Virgo Collaboration], GCN Circular 24098 (2019).

[21] D.Chatterjee [LIGO Scientific Collaboration and Virgo Collaboration], GCN Circular 24141 (2019).

[22] S.Ghosh [LIGO Scientific Collaboration and Virgo Collaboration], GCN Circular 24377 (2019).

[23] D.Chatterjee [LIGO Scientific Collaboration and Virgo Collaboration], GCN Circular 24503 (2019).

[24] M.Drago [LIGO Scientific Collaboration and Virgo Collaboration], GCN Circular 24522 (2019).

[25] S.Ghosh [LIGO Scientific Collaboration and Virgo Collaboration], GCN Circular 24570 (2019).

[26] R.De Pietri [LIGO Scientific Collaboration and Virgo Collaboration], GCN Circular 24598 (2019).

[27] G.Mo [LIGO Scientific Collaboration and Virgo Collaboration], GCN Circular 24621 (2019).

[28] S.J.Kapadia [LIGO Scientific Collaboration and Virgo Collaboration], GCN Circular 24632 (2019).

[29] P.Shawhan [LIGO Scientific Collaboration and Virgo Collaboration], GCN Circular 24717 (2019).

[30] A.Aab [Pierre Auger Collaboration], arXiv preprint (2019) arXiv:1906.07419, prepared for submission to JCAP.

[31] G.J.Feldman, R.D.Cousins, Phys. Rev. D 57 (1998) 3873.

[32] A.Aab [Pierre Auger Collaboration], Phys. Rev. D 94 (2016) 122007.

[33] P.Padovani, P.Giommi, E.Resconi, T.Glauch, B.Arsioli, N.Sahakyan, M.Huber, MNRAS 480 (2018) 192. 